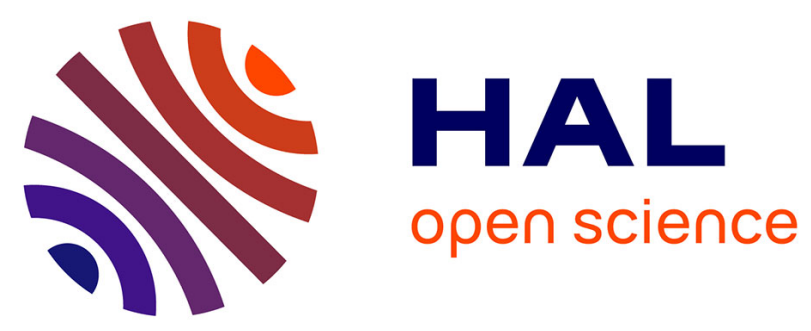

\title{
HDR-ARtiSt: An Adaptive Real-time Smart camera for High Dynamic Range imaging
}

Pierre-Jean Lapray, Barthélémy Heyrman, Dominique Ginhac

\section{To cite this version:}

Pierre-Jean Lapray, Barthélémy Heyrman, Dominique Ginhac. HDR-ARtiSt: An Adaptive Real-time Smart camera for High Dynamic Range imaging. Journal of Real-Time Image Processing, 2016, 12 (4), pp.747-762. 10.1007/s11554-013-0393-7 . hal-00794471v3

\section{HAL Id: hal-00794471 \\ https://hal.science/hal-00794471v3}

Submitted on 14 Jan 2014

HAL is a multi-disciplinary open access archive for the deposit and dissemination of scientific research documents, whether they are published or not. The documents may come from teaching and research institutions in France or abroad, or from public or private research centers.
L'archive ouverte pluridisciplinaire HAL, est destinée au dépôt et à la diffusion de documents scientifiques de niveau recherche, publiés ou non, émanant des établissements d'enseignement et de recherche français ou étrangers, des laboratoires publics ou privés. 


\title{
HDR-ARtiSt: An Adaptive Real-time Smart camera for High Dynamic Range imaging
}

\author{
Received: date / Revised: date
}

\begin{abstract}
This paper describes a complete FPGA-based smart camera architecture named HDR-ARtiSt (High Dynamic Range Adaptive Real-time Smart camera) which produces a realtime High Dynamic Range (HDR) live video stream from multiple captures. A specific memory management unit has been defined to adjust the number of acquisitions to improve HDR quality. This smart camera is built around a standard B\&W CMOS image sensor and a Xilinx FPGA. It embeds multiple captures, HDR processing, data display and transfer, which is an original contribution compared to the state of the art. The proposed architecture enables a real-time HDR video flow for a full sensor resolution (1.3 Mega pixels) at 60 frames per second.
\end{abstract}

\section{Introduction}

Standard cameras capture only a fraction of the information that is visible to the human eye. This is specifically true for natural scenes including areas of low and high illumination due to transitions between sunlit and shaded areas. When capturing such a scene, many cameras are unable to store the full Dynamic Range (DR) resulting in low quality video where details are concealed in shadows or washed out by sunlight. High Dynamic Range (HDR) imaging techniques appear as a solution to overcome this issue by encoding digital video with higher than standard 24-bit RGB format, and then increasing the range of luminance that can be stored. Reinhard et al [36] provide an exhaustive review, highlighting various fields of applications of HDR cameras. For example, HDR capturing techniques are essential for outdoor security applications in which unpredictable illumination changes may affect performance algorithms $[5,28]$.

Pierre-Jean Lapray, Barthélémy Heyrman and Dominique Ginhac are with the Laboratory of Electronic, Computing and Imaging Sciences, Le2i UMR 6306, Univ Burgundy, Dijon, France.

Dominique Ginhac

Le2i UMR 6306, Univ Burgundy, Dijon, France

Tel.: +333803938 86

E-mail: dginhac@u-bourgogne.fr
Similarly, HDR techniques should facilitate object tracking or automotive applications [40, 43, 24] under uncontrolled illumination. Recent research programs on machine vision have clearly demonstrated the benefits of real-time HDR vision [20,38]. Finally, medical applications require high precision images and HDR lighting techniques to improve the rendering quality of imaging systems $[25,17]$.

There are two major approaches to create HDR content: to develop new HDR sensors or to combine multiple exposure frames captured by conventional Low Dynamic Range (LDR) sensors. First, several HDR sensors have been designed with techniques such as well-capacity adjusting, timeto-saturation, or self-reset (see [22] for a comparative analysis). Most of these sensors are tailor-made and provide dedicated processing units to extend DR [1, 39, 7, 26, 30, 12].

The second method relies on conventional off-the-shelf LDR sensors to capture the HDR data by recording multiple acquisitions of the same scene while varying the exposure time $[9,29,37,21]$. By limiting the exposure time, the image loses low-light detail in exchange for improved detail in bright areas. By increasing the exposure time, the image is only detailed in the dark areas because of the pixel saturation in bright areas. Each pixel is at least properly exposed in one image and is under or overexposed in other images of the sequence. The images are then combined into a single HDR frame (i.e. a radiance map). Finally, since current display technology has a limited DR, HDR images need to be compressed by tone mapping operators [10, 33, 6, 2, 3, 27] in such a way that the visual sensation of the real scene is faithfully reproduced, as depicted in Fig. 1.

This paper presents a complete FPGA-based smart camera architecture named HDR-ARtiSt (High Dynamic Range Adaptive Real-time Smart camera). This smart camera is able to provide a real-time HDR live video from multiple exposure capturing to display, through radiance maps and tone mapping. The main contribution of this work is the generation of a new FPGA embedded architecture producing an uncompressed B\&W $1280 \times 1024$-pixel HDR live video at 60 fps. An embedded DVI controller is also provided to display this HDR live video on a standard LCD monitor. The HDRARtiSt camera could obviously embed some complex im- 

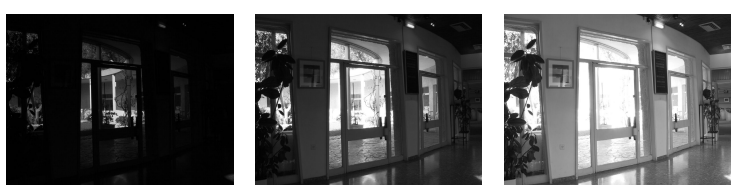

(a) Low exposure (b)

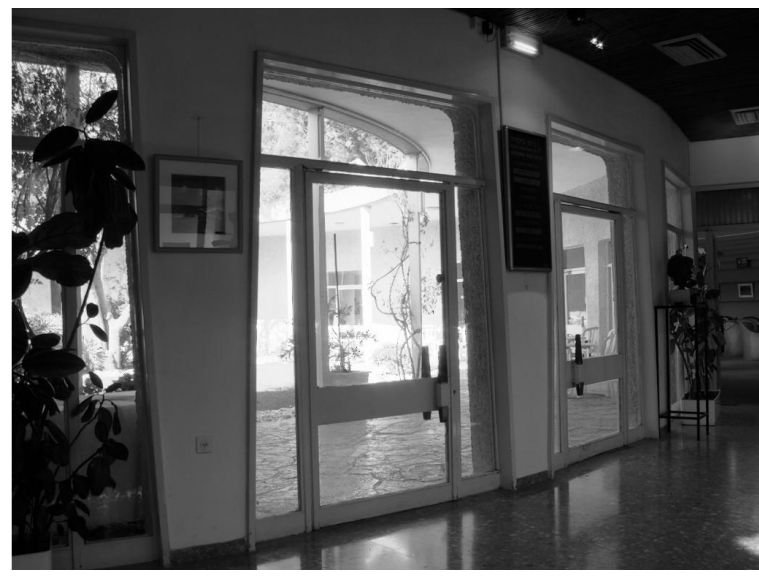

(d) HDR image

Fig. 1 HDR frame of a real scene captured with 3 different exposure times (sequences by Fattal et al [14]).

age processing applications onto the FPGA or could be connected to a more standard PC managing the video stream.

The remainder of the paper is as follows: in Section 2, we briefly review the literature on existing HDR systems. Section 3 describes our proposed hardware architecture, highlighting the multi-streaming memory management unit designed to address the computation capacity and memory bandwidth limitations. A first implementation using a two-frame acquisition is presented in Section 4. Based on a detailed study of the visual quality of this implementation, an improved three-frame solution is described in Section 5. Finally, Section 6 concludes this paper and outlines directions for future work.

\section{Related work}

The problems of capturing the complete dynamic range of a real scene and reducing this dynamic range to a viewable range have drawn the attention of many authors. However, the main part of the proposed algorithms have been developed without taking into account the specifications and the difficulties inherent to hardware implementations. Unfortunately, these works are not generally suitable for efficient real-time implementation on smart cameras. As a consequence, generating real-time HDR live video remains an interesting challenge.

\subsection{Existing hardware architectures}

In 2007, Hassan and Carletta [19] described an FPGA-based architecture for local tone mapping of gray scale HDR images, able to generate $1,024 \times 768$-pixel image at 60 frames per second. The architecture is based on a modification of the nine-scale [34] operator. Several limitations can be noticed. Firstly, this work focuses only on the tone-mapping process and does not care about the HDR capture, using a set of high dynamic range still images from the Debevec library [9]. Secondly, the tone mapping operator requires to store a full image before evaluating the logarithmic average of the image, leading to a video latency. This limitation can be overcome by using the logarithmic average of the previous image to normalize the current image. Finally, using the Gaussian pyramid requires a lot of bits per pixel, increasing the amount of onboard memory. Another real-time hardware implementation of tone mapping has been recently proposed by Vytla et al [48]. They use the Fattal et al [14] local algorithm. This operator is less complex than the Reinhard's operator, then requiring less onboard memory. The key point of this work is the inexpensive hardware implementation of a simplified Poisson solver for Fattal's operator. It gives a realtime tone mapping implementation on a Stratix II FPGA operating at 100 frames per second with one megapixel image resolution.

Chiu et al [8] describe a methodology for the development of a tone-mapping processor of optimized architecture using an ARM SOC platform, and illustrate the use of this novel HDR tone-mapping processor for both photographic and gradient compression. Based on this methodology, they develop an integrated photographic and gradient tone-mapping processor that can be configured for different applications. This newly-developed processor can process $1,024 \times 768$-pixel images at $60 \mathrm{fps}$, runs at $100 \mathrm{MHz}$ clock and consumes a core area of $8.1 \mathrm{~mm}^{2}$ under a TSMC 0.13 $\mu \mathrm{m}$ technology.

Kang et al [21] describe an algorithmic solution computing both video capture and HDR synthesis, and able to generate HDR video from an image sequence of a dynamic scene captured while varying the exposure at each frame (alternating light and dark exposures). For this purpose, the approach consists of three main parts: automatic exposure control during capture, HDR stitching across neighbouring frames, and tone mapping for viewing. The implemented technique produces video with increased dynamic range while handling moving parts in the scene. However, the implementation on a $2 \mathrm{GHz}$ Pentium 4 machine does not reach the real-time constraint because the processing time for each $1,024 \times 768$-pixel video frame is about 10 seconds $(8 \mathrm{sec}-$ onds for the radiance mapping and 2 seconds for the tone mapping). Based on Kang's algorithms, Youm et al [52] create an HDR video by merging two images from different exposures acquired by a stationary video camera system. Their methodology mainly relies on the simple tactic of automatically controlling exposure times and effectively combines bright and dark areas in short and long exposure frames. 
Unfortunately, they do not reach real-time processing with about 2.5 seconds for each $640 \times 480$-pixel frame on a 1.53 GHz AMD Athlon machine.

Finally, Ke et al [23] propose an innovative method to generate HDR video. This method differs drastically with the above-mentioned state-of-the-art works because only one LDR image is enough to generate HDR-like images, with fine details and uniformly-distributed intensity. To obtain such a result, they implement a hardware-efficient virtual HDR image synthesizer that includes virtual photography and local contrast enhancement. Under a UMC 90-nm CMOS technology, it achieves real-time for $720 \times 480$-pixel video frames at $60 \mathrm{fps}$.

\subsection{Efficient algorithms for HDR video}

Generating an HDR live video stream as well as HDR still images consists of automatically determining the optimal exposures for multiple frames capture, computing radiance maps, and local/global tone mapping for viewing on a standard LCD monitor.

Table 1 is the list of common terms and variables used in the equations of the following sections.

Table 1 List of commonly used terms and variables.

\begin{tabular}{|c|c|}
\hline $\mathrm{P}$ & Number of frames to create one HDR frame \\
\hline$I_{p}$ & $p$ th frame in the sequence of $P$ frames \\
\hline$M$ & Frame height (i.e. row number) \\
\hline$N$ & Frame width (i.e. column number) \\
\hline$Z_{i j}^{p}$ & Luminance of the pixel $(i, j)$ in the $p$ th frame \\
\hline$E_{i j}$ & Luminance (or radiance) of the HDR pixel $(i, j)$ \\
\hline$g$ & Camera transfer function $(\mathrm{CTF})$ \\
\hline$\Delta t_{p}$ & Exposure time of the $p$ th frame \\
\hline$D_{i j}$ & Tone mapped pixel \\
\hline$D_{m i n}, D_{\max }$ & Minimum and maximum values of the display devices \\
\hline$\tau$ & Overall brightness of the mapped frame \\
\hline$L_{m}$ & Line index \\
\hline$W \& R$ & Memory Write and Read operations \\
\hline
\end{tabular}

\subsubsection{Step 1: HDR creating}

Digital cameras have limited dynamic range since they can only capture from 8-bit to 14-bit images, mainly due to the limitations of the analog to digital converters in terms of noise levels. The most common method to generate HDR content is to capture multiple images of a same scene with different exposure times. If the camera has a linear response, we can easily recover the HDR luminance $E_{i j}$ from each luminance $Z_{i j}^{p}$ and exposure times $\Delta t_{p}$ stored in each frame $p$. Unfortunately, cameras do not have a linear response (i.e. $Z_{i j}^{p}$ is not proportional to $E_{i j}$ and $\Delta t_{p}$ ), and we have to estimate the non-linear camera transfer function (CTF) called $g$ to combine properly the different exposures.
Three popular algorithms for recovering this camera transfer function can be extracted from literature: Debevec and Malik [9], Mitsunaga and Nayar [29], and Robertson et al [37]. According to the detailed description of these methodologies and the comparison of their real-time software implementations, we decided to use the Debevec's method. The main advantage of this approach is that there is very little constraint about the response function (other than its invertability). Moreover, the proposed algorithm has proved to be quite robust and easy to use, due to the simplicity of equations $[18,15]$. The CTF function is evaluated from the film reciprocity equation $f$ :

$Z_{i j}^{p}=f\left(E_{i j} \Delta t_{p}\right)$

The CTF function $g$ is defined as $g=\ln f^{-1}$ and can be obtained by minimizing the following function:

$$
\mathscr{O}=\sum_{i=1}^{M} \sum_{j=1}^{N} \sum_{p=1}^{P}\left[g\left(Z_{i j}^{p}\right)-\ln E_{i j}-\ln \Delta t_{p}\right]^{2}+\lambda \sum_{z=Z_{\min }+1}^{Z_{\max }-1} g^{\prime \prime}(z)^{2}
$$

Where $\lambda$ is a weighting scalar depending on the amount of noise expected on $g, Z_{\min }$ and $Z_{\max }$ are respectively the lowest and the greatest pixel values. The evaluation of $g$ only requires the evaluation of a finite number of values between $Z_{\min }$ and $Z_{\max }$ (typically 1,024 values for a 10-bit precision sensor). These values can be preliminary evaluated from a sequence of several images, then stored in the camera, and reused further to convert pixel values. For recovering the HDR radiance value of a particular pixel, all the available exposures of this pixel are combined using the following equation [42]:

$\ln E_{i j}=\frac{\sum_{p=1}^{P} \omega\left(Z_{i j}^{p}\right)\left[g\left(Z_{i j}^{p}\right)-\ln \Delta t_{p}\right]}{\sum_{p=1}^{P} \omega\left(Z_{i j}^{p}\right)}$

Where $\omega(z)$ is a weighting function giving higher weight to values closer to the middle of the function:

$\omega(z)=\left\{\begin{array}{l}z-Z_{\min } \text { for } z \leq \frac{1}{2}\left(Z_{\min }+Z_{\max }\right) \\ Z_{\max }-z \text { for } z>\frac{1}{2}\left(Z_{\min }+Z_{\max }\right)\end{array}\right.$

Originally, the Debevec's method has been developed for photography but according to G. Yourganov [15], this method can be easily applied to digital video, both for static and dynamic scenes, if captures are fast enough that light changes can be safely ignored. Consequently, such a method is widely used to produce HDR video, by capturing frames with alternating bright and dark exposures, as pointed by Tocci et al [47]. 


\subsubsection{Step 2: Tone mapping}

The HDR pixels are represented by a high bit-depth conflicting with the standard display devices, requiring a high to low bit-depth tone mapping. Cadík et al [6] show that the global part of a tone mapping operator is essential to obtain goods results. A psychophysical experiment by Yoshida et al [51], based on a direct comparison among the appearances of real-world HDR images shows that global methods like F. Drago and Chiba [13] or Reinhard et al [35] are perceived as the most natural ones. Moreover, a global tone mapper is the easiest way to reach real-time constraints because local operators require more complex computations. The choice of a candidate tone mapping operator has been done after comparing and intensively testing several $\mathrm{C}++$ global algorithms applied to a radiance map constructed from two or three images. Results are provided in Table 2. According to our temporal and hardware constraints, the best compromise is the global tone mapper from Duan et al [11]. As an illustration, the HDR image depicted on the Fig. 1 has been obtained with this algorithm. This tone mapper compresses the luminance of the HDR pixel $E_{i j}$ to a displayable luminance $D_{i j}$ with the equation:

$$
D_{i j}=C *\left(D_{\max }-D_{\min }\right)+D_{\min }
$$

with $C=\frac{\ln \left(E_{i j}+\tau\right)-\ln \left(E_{i j(\min )}+\tau\right)}{\ln \left(E_{i j(\max )}+\tau\right)-\ln \left(E_{i j(\min )}+\tau\right)}$

where $E_{i j(\min )}$ and $E_{i j(\max )}$ are the minimum and maximum luminance of the scene and $\tau$ is inversely linked to the brightness of the mapped image. Increasing $\tau$ makes darker images while lower values give brighter images.

\begin{tabular}{lrrrrr}
\hline TMO & PSNR & UQI & SSIM & NRMSE & Time(s) \\
\hline Drago [13] & 34.93 & 0.54 & 0.58 & 0.1 & 5.43 \\
Duan [11] & 42.34 & 0.97 & 0.91 & 0.01 & 5.71 \\
Reinhard [35] & 29.11 & 0.89 & 0.8 & 0.32 & 5.52 \\
Schlick [41] & 40.71 & 0.43 & 0.56 & 0.03 & 5.50 \\
Tumblin [32] & 42.01 & 0.14 & 0.29 & 0.02 & 5.59 \\
\hline
\end{tabular}

Table 2 Comparison metrics for TMOs algorithms applied to a common HDR image (memorial by Debevec) constructed from three images. Exposure times used are $32 \mathrm{~s}, 1 \mathrm{~s}$ and $31,25 \mathrm{~ms}$.

\section{Architecture of the HDR-ARtiSt platform}

To compute real-time HDR algorithms, a dedicated FPGAbased smart camera architecture was designed to address the computation capacity and memory bandwidth requirement (see Fig. 2 for an overview). This architecture does not put any restriction on the number of frames used for HDR creating. In the remainder of this paper, this generic architecture will be shortly called HDR-P, where $P$ is the number of frames.

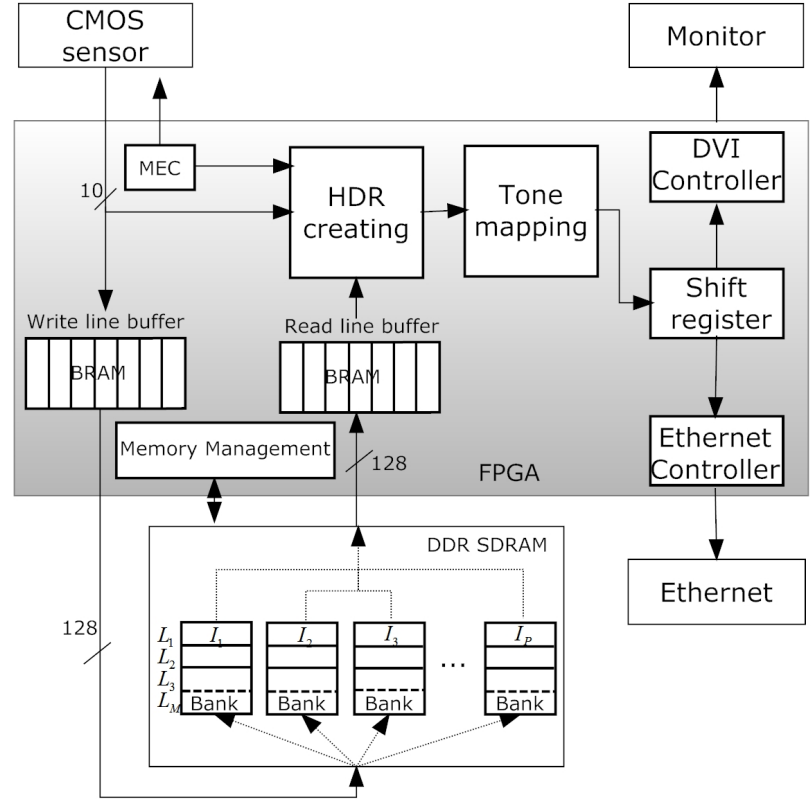

Fig. 2 Overview of the HDR-P video system architecture.

\subsection{Global hardware architecture}

The HDR-ARtiSt platform is a smart camera built around a Xilinx ML507 board, equipped with a Xilinx Virtex-5 XC5VFX70T FPGA (see Fig. 3). The motherboard includes a 256 MB DDR2 SDRAM memory used to buffer the multiple frames captured by the sensor. Several industry-standard peripheral interfaces are also provided to connect the system to the external world. Among these interfaces, our vision system implements a DVI controller to display the HDR video on a LCD monitor. It also implements an Ethernet controller to store frames on a host computer.

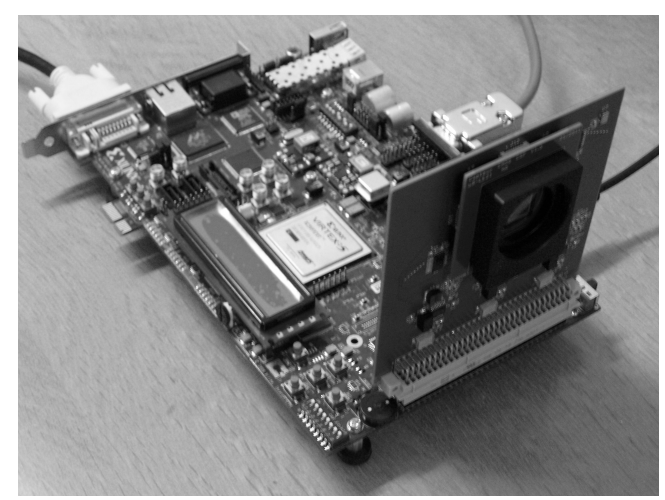

Fig. 3 HDR-P video hardware is prototyped on a Xilinx Virtex-5 ML507 FPGA board and a daughter card with the EV76C560 1.3Mpixel sensor.

A custom-made PCB extension board has been designed and plugged into the FPGA board to support the Ev76c560 image sensor, a 1,280 $\times 1,024$-pixel CMOS sensor from e $2 \mathrm{v}$ 
[46]. It offers a 10-bit digital readout speed at $60 \mathrm{fps}$ in full resolution. It also embeds some basic image processing functions such as image histograms, evaluation of the number of low and high saturated pixels. Each frame can be delivered with results of these functions encoded in the video data stream header.

\subsection{Multi-streaming Memory Management Unit}

Standard HDR techniques require two sequential steps: 1) $P$ single frames must be captured and stored into memory, and 2) the HDR frame can be computed. The main drawback is the limited output framerate. As illustrated on Fig. 4, with $P=3$ single frames (low, middle and high exposures) captured at $60 \mathrm{fps}$, the resulting HDR video is dramatically limited to 20 fps: the first HDR frame $H_{1}$ is computed from the frames $I_{1}, I_{2}$, and $I_{3}$, the second HDR frame $H_{2}$ from $I_{4}$, $I_{5}$, and $I_{6}$.

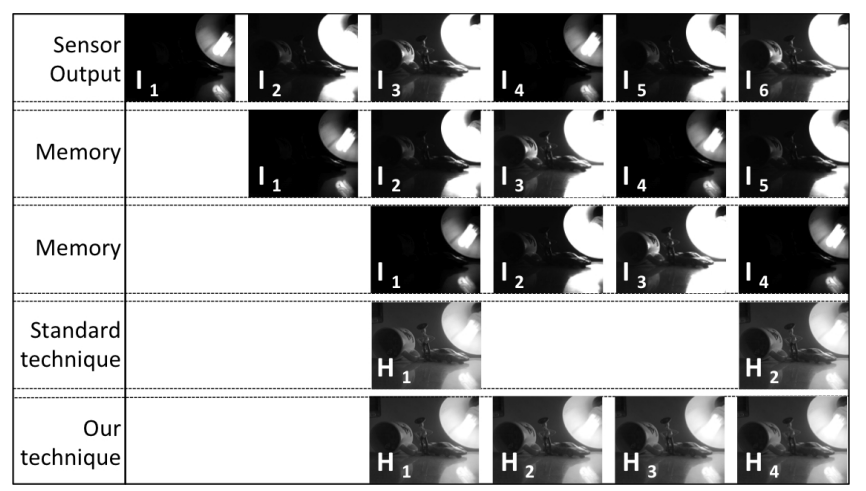

Fig. 4 Framerates of the HDR standard technique and our technique.

To overcome this limitation, we propose a specific Memory Management Unit (MMU) able to continuously build a new HDR frame at the sensor framerate from the $P$ previous frames. As seen on Fig. 4, the HDR frame $\mathrm{H}_{2}$ is built from the frames $I_{2}, I_{3}$, and $I_{4}$; the HDR frame $H_{3}$ from $I_{3}$, $I_{4}$, and $I_{5}$, etc. This multi-streaming MMU (called MMUP, with $P$ the number of frames) continuously manages the storage of $P-1$ frames, the oldest frame being systematically replaced with the new acquired frame. Simultaneously, the MMU-P manages the reading of these $P-1$ frames with the sensor output in order to feed the HDR creating process. For this purpose, a time sharing strategy is used to store and read back the different video streams into the DDR2 memory. Small size buffers implemented in FPGA embedded Block RAMs (BRAMs) are required to store temporary data and to handle the sharing of SDRAM data bus. To meet the challenge of real-time constraints and to minimize the buffer sizes, the MMU-P performs row-by-row read/write operations to transfer the different streams.

Before any HDR computation, the memory unit needs to be set up (see Fig. 5). Indeed, when the vision system has just been turned on, an initialization step captures and stores row-by-row $P-1$ frames into the SDRAM $\left(W L_{m} I_{p}\right.$ with $0<$ $m \leq M$ and $0<p<P$ ). After receiving the last row $L_{M}$ of the frame $I_{P-1}$, the first rows $L_{1}$ of each previously stored frame $\left(R L_{1} I_{p}\right.$ with $\left.0<p<P\right)$ are read between frames and buffered into BRAMs to be quickly available for the HDR creating process.

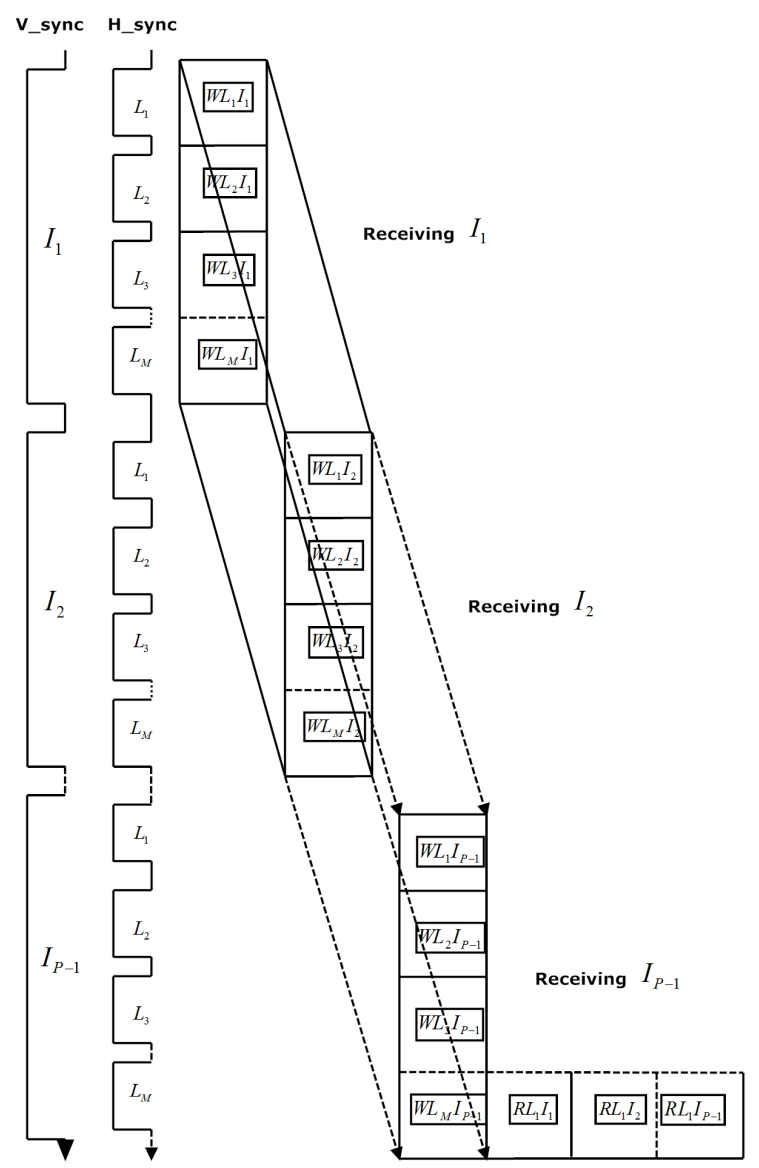

Fig. 5 Initialization of the Memory Management Unit with storage of the first $P-1$ th frames.

The second step is managed by the MMU-P core and starts at the beginning of the $p$ th capture. During each row interval, the current row $L_{m}$ is written to the SDRAM memory while rows $L_{m+1}$ of the $P-1$ previous frames are read and buffered into BRAMs as depicted in Fig. 6. With such a technique, when the sensor delivers a new row, the HDR creating process has a simultaneous and easy access to the current row and the corresponding rows of the previous frames. Then, the first HDR frame is obtained at the end of the capture of the frame $I_{p}$ at $t=t_{H D R_{1}}$ (see Fig. 6). This process is then reiterated with the frame $I_{p+1}$ and the computation of the second HDR frame from $I_{2}, I_{3}, \ldots, I_{p+1}$ at $t=t_{H D R_{2}}$, etc.

To summarize, the MMU-P is able to capture and store the current stream of pixels from the sensor, and delivers simultaneous $P-1$ pixel streams previously stored to the HDR creating process. With such a memory management, 


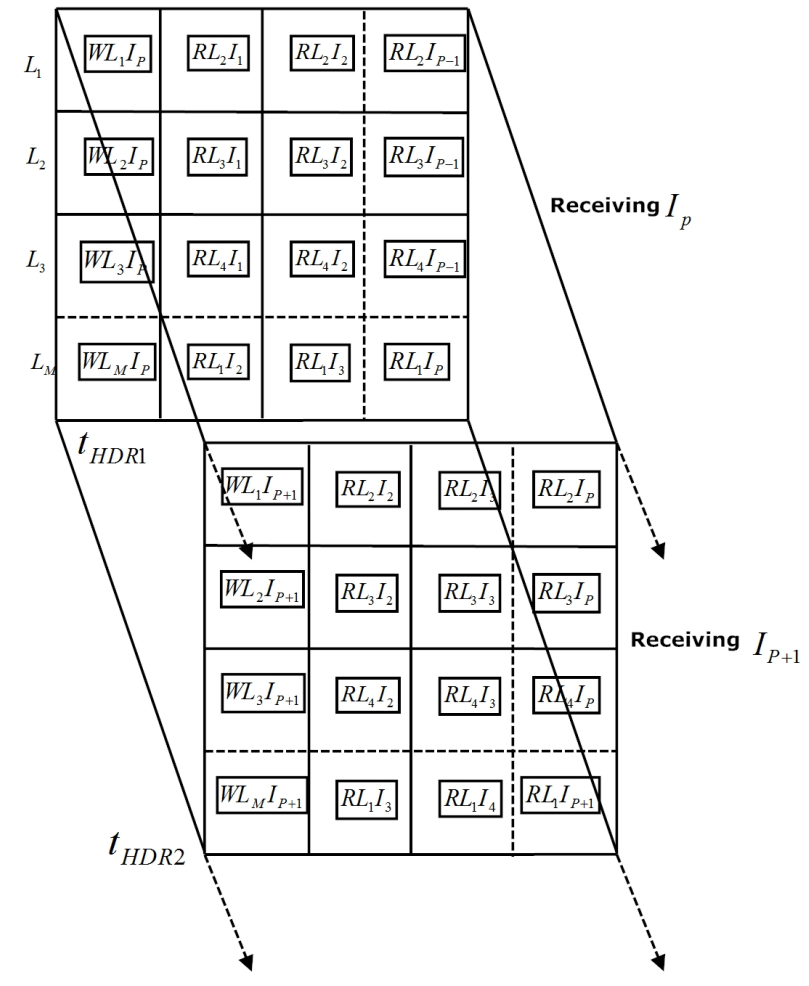

Fig. 6 Memory Management Unit with parallel acquisition of new lines of pixels and reading of previously stored lines.

we avoid waiting for the capturing of new $P$ frames before computing any new HDR data. Once the initialization is done, our system is synchronized with the sensor framerate (i.e. $60 \mathrm{fps}$ ) and is able to produce a new HDR frame for each new capture. Moreover, in terms of memory, the MMU-P requires to store only $P-1$ frames, because the oldest captured frame is read and overwritten by the current frame acquired by the sensor. For reasons of efficiency, the MMU-P reads and stores lines of pixels. The computation of an HDR line requires $P$ memory accesses: one write operation for the current line captured by the sensor and $P-1$ read operations for the $P-1$ lines previously stored in memory during the row interval. On the opposite, the traditional technique requires to store $P$ frames in memory. It requires at least $P+1$ memory accesses (one write and $P$ read operations). Finally, to generate an HDR image, the MMU-P saves $M$ writing memory access operations (with $M$, the line number of each frame).

\section{Implementation of the HDR-2 video system}

The HDR-P has been first prototyped on the HDR-ARtiST platform with the limitation $P=2$, using only 2 frames to generate each HDR frame.

\subsection{Multiple Exposure Control}

The auto exposure bracketing system implemented in digital cameras is a very useful technique to automatically capture multiple exposures at fixed multiples around an optimum exposure. Our Multiple Exposure Control (MEC) for low and high exposure images is slightly different because our objective is rather to evaluate each individual exposure to get the maximum of well-exposed pixels. Our work is relatively close to the Kang's exposure control [21] and the Alston's double-exposure system [4]. In our case, the exposure settings alternate between two different values that are continuously adapted to reflect the scene changes. Following Gelfand et al [16], we require that fewer than $10 \%$ of the pixels are saturated at high-level for the short exposure frame. If too many pixels are bright, the exposure time is decreased for the subsequent short exposure captures. Similarly, we require that fewer than $10 \%$ pixels are saturated at low-level for the long exposure. If too many pixels are dark, the exposure time is increased. Gelfand et al. stop this iterative process when the two exposures are stable, and use these values for the capture of the full resolution photography. Such an approach is optimal to capture a single HDR image but can not be considered for an HDR video.

In our approach, we decide to continuously update the set of exposure times from frame to frame in order to minimize the number of saturated pixels by instantaneously handling any change of the light conditions. The estimation of the best exposure times is computed from the 64-level histogram $(q)$ provided automatically by the sensor in the datastream header of each frame. For each low exposure frame $\left(I_{L}\right)$ and each high exposure $\left(I_{H}\right)$, we evaluate $Q_{L}$ and $Q_{H}$ that are respectively the ratio of pixels in the four lower levels and the four higher levels of the histogram:

$$
Q_{L}=\sum_{h=1}^{h=4} \frac{q(h)}{N} \quad Q_{H}=\sum_{h=60}^{h=64} \frac{q(h)}{N}
$$

where $q(h)$ is the amount of pixels in each histogram category $h$ and $N$ the total number of pixels. A series of decisions can be performed in order to evaluate the low exposure time $\left(\Delta t_{L, t+1}\right)$ and the high exposure time $\left(\Delta t_{H, t+1}\right)$ at the next iteration $(t+1)$ of the acquisition process.

$$
\begin{gathered}
\Delta t_{L, t+1}=\left\{\begin{array}{l}
\Delta t_{L, t}+10 x \text { if } Q_{L}>Q_{L, r e q}+t h r_{L p} \\
\Delta t_{L, t}-10 x \text { if } Q_{L}<Q_{L, r e q}-t h r_{L p} \\
\Delta t_{L, t}+1 x \text { if } Q_{L}>Q_{L, r e q}+t h r_{L m} \\
\Delta t_{L, t}-1 x \text { if } Q_{L}<Q_{L, r e q}-t h r_{L m}
\end{array}\right. \\
\Delta t_{H, t+1}=\left\{\begin{array}{l}
\Delta t_{H, t}-10 x \text { if } Q_{H}>Q_{H, r e q}+t h r_{H p} \\
\Delta t_{H, t}+10 x \text { if } Q_{H}<Q_{H, r e q}-t h r_{H p} \\
\Delta t_{H, t}-1 x \text { if } Q_{H}>Q_{H, r e q}+t h r_{H m} \\
\Delta t_{H, t}+1 x \text { if } Q_{H}<Q_{H, r e q}-t h r_{H m}
\end{array}\right.
\end{gathered}
$$

Where $x$ is the integration time of one sensor row. $Q_{L, r e q}$ and $Q_{H, r e q}$ are respectively the required number of pixels 
for the low levels and the high levels of the histogram. In order to converge to the best exposure times as fast as possible, we decide to use two different thresholds for each exposure time: $t h r_{L m}$ and $t h r_{L p}$ are thresholds for the low exposure time whereas $t h r_{H m}$ and $t h r_{H p}$ are for the high exposure time. In our design, values of $Q_{L, r e q}$ and $Q_{H, \text { req }}$ are fixed to $10 \%$ pixels of the sensor. Values of $t h r_{L m}$ and $t h r_{H m}$ are fixed to $1 \%$ whereas $t h r_{L p}$ and $t h r_{H p}$ are fixed to $8 \%$.

Fig. 7 illustrates how the MEC algorithm automatically estimates the new exposure times while rapidly varying illumination in the scene. When switching on the lamp, the number of white saturated pixels significantly increases (15.3 $\%$ for Fig. 7.a). So, the MEC rapidly decreases the exposure time to get less than $10 \%$ saturated pixels for the high exposure time $\Delta t_{3}(5.4 \%$ for Fig. 7.a). A similar approach is used in order to estimate the low exposure time $\Delta t_{1}$.

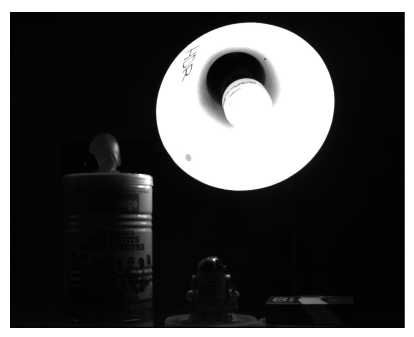

(a) Before MEC.

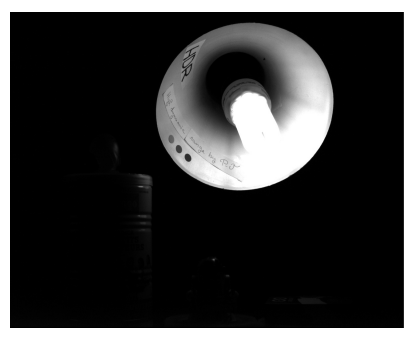

(b) After MEC.
Fig. 7 Figure (a) has $15.3 \%$ of white saturated pixels when the lamp is switched on. After MEC, Figure (b) has $5.4 \%$ of white pixels.

\subsection{Memory interface implementation}

The HDR-ARtiST platform embeds a $256 \mathrm{MB}$ memory with a 128-bit wide interface. Memory access operations are managed by a custom SDRAM controller specifically generated by the Xilinx Memory Interface Generator. The operation frequency of the SDRAM has been fixed to $125 \mathrm{MHz}$. The e2v sensor captures $60 \mathrm{fps}$ with an inter-frame time of $236 \mu \mathrm{s}$ and a row time of $14.1 \mu \mathrm{s}(10 \mu \mathrm{s}$ for the 1,280 pixels and an inter-row time of $4.1 \mu \mathrm{s}$ ). BRAMs are used on input and output of the SDRAM, and act as data row buffers to support DDR read and write burst operations. A set of $P$ different BRAMs is required: one BRAM used to feed the SDRAM with the incoming sensor data and a set of $P-1$ BRAMs supplied with the parallel streams of the previously captured frames. These block memories manage independent clocks and support non-symmetric aspect ratios for IO operations. Each BRAM is 2048 deep and 10-bit wide, in order to manage a full 1,280-pixel row. A full row of 1,280 pixels captured by the sensor is written into the memory in $\frac{1,280 \times 10}{128 \times 125 \cdot 10^{6}}=$ $0.8 \mu \mathrm{s}$. Similarly, the time needed for reading a 1,280-pixel row from the SDRAM is identical. These two operations are low time-consuming and take place during the inter-row interval.
4.3 Algorithm simplifications for an efficient hardware implementation

In order to meet both the temporal constraints and platform requirements, some simplifications of the algorithms described in Section 2.2 are proposed.

\subsubsection{HDR creating module}

The evaluation of the CTF function $g$ has not been implemented on the platform because it needs to be computed only once. So, the parameters of the CTF are preliminary evaluated by a dedicated PC software from a sequence of images, and then stored into a Look-Up Table (LUT, 1024word memory) on the FPGA. Moreover, in order to reduce the computation complexity and to optimize the hardware resources, some other mathematical operations, such as neperian logarithms, are also pre-calculated and registered in LUTs.

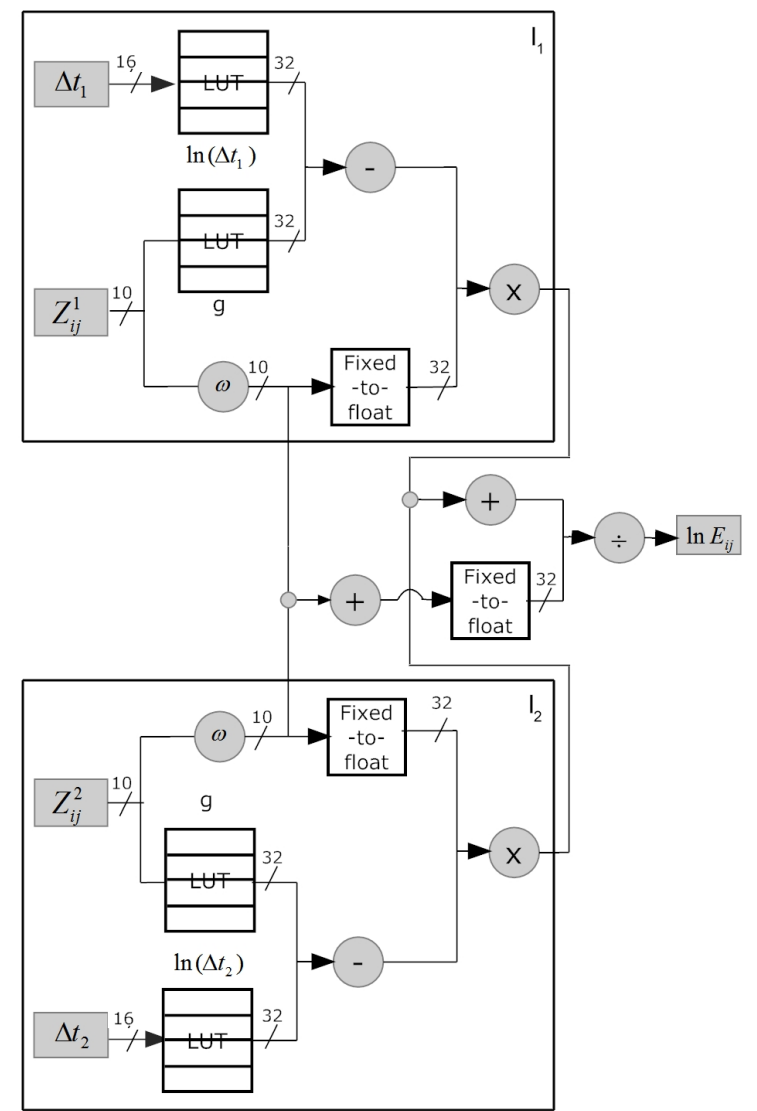

Fig. 8 HDR creating pipeline for HDR-2 video, using LUTs tree.

Finally, the hardware cost of the implementation of the HDR creating described in Eq. 3 only depends of the number of bracketed frames used to create one HDR video frame. (see Fig. 8 for HDR-2). Each frame requires two 32-bit arithmetic operators (1 subtractor, 1 multiplier) and one transition from 10-bit to IEEE754 32-bit wide (Fixed-to-Float). Other 
hardware resources (a 10-bit adder, a 32-bit adder, a 32-bit divider and a Fixed-to-Float operator) are also required, independently of the number of frames. A pipeline structure is implemented to speed-up the processing.

Table 3 compares the hardware resources required for the arithmetic operators used in the HDR pipeline. It reveals that a 32-bit floating-point format requires significantly less resource, specifically for complex operations such as division, square root and even multiplication. Only addition and subtraction are better in fixed-point format. Since division and multiplication are common operations in our design, we decided to use floating-point format.

\begin{tabular}{|l|c|c|c|c|}
\hline Operator & \multicolumn{2}{|c|}{ Fixed point } & \multicolumn{2}{c|}{ Floating point } \\
\hline & LUTs & DSPs & LUTs & DSPs \\
\hline Add/sub & 75 & 0 & 477 & 0 \\
\hline & 0 & 1 & 287 & 2 \\
\hline \hline Mult. & 696 & 0 & 659 & 0 \\
\hline & 132 & 1 & 107 & 3 \\
\hline \hline Divide 1 Cycle & 1377 & 0 & 780 & 0 \\
\hline Divide 25 Cycles & - & - & 187 & 0 \\
\hline Square root 1 Cycle & 1550 & 0 & 533 & 1 \\
\hline Square root 25 Cycles & - & - & 170 & 1 \\
\hline
\end{tabular}

Table 3 Comparison of resource estimations for arithmetic operators (Xilinx IPs) in fixed-point and floating-point format.

\subsubsection{Tone mapping module}

The hardware implementation of the Eq. 5 requires the preliminary estimation of the terms $E_{i j(\min )}$ and $E_{i j(\max )}$ relative to the HDR frame before any tone mapping operation. However, as our hardware pipeline computes HDR imaging and tone mapping on the incoming pixels stream, such an approach is inconceivable. We make the assumption that the light conditions do not vary significantly between two consecutive frames captured at $60 \mathrm{fps}$. So, these two terms are computed from the current HDR frame (CMP Min and CMP Max operators), stored in registers (Reg) and then used as the Min/Max values for the tone mapping of the next HDR frame, as seen in Fig. 9. $\tau$ controls the overall brightness of the mapped frame. According to Duan et al [11], a larger $\tau$ makes the mapped frame darker and smaller $\tau$ makes the mapped frame brighter. Several values have been tested in simulation and we decide to set $\tau=0$ because it simplifies the hardware implementation. We also set $D_{\max }=255$ and $D_{\min }=0$ since standard monitors use a 8-bit format for each channel. The multiplication by 255 is easily done by adding 8 to the exponent in floating point. This may change in the future due to successful efforts in building higher resolution displays. Our design will be able to follow this evolution because tone mapped frames with higher resolutions could be easily produced by increasing $D_{\max }$ and changing the final Float-to-Fixed operator.

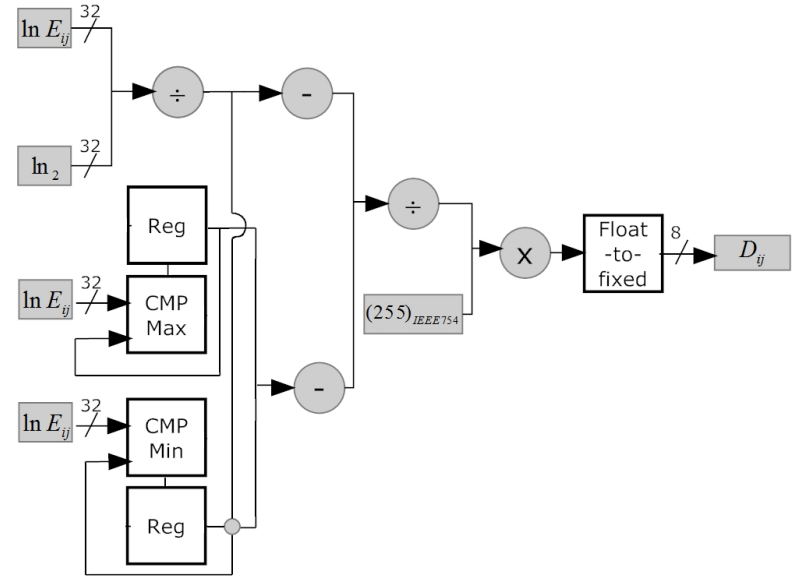

Fig. 9 Tone mapping computation.

\subsection{Results}

Simulation and validation phases of the HDR-2 architecture have been made using Modelsim. Then, it has been implemented on the HDR-ARtiST platform. Table 4 is a summary of the whole design and Table 5 describes details of the synthesis report. Usually, FPGA-based image processing requires many specific devices such as SRAM memory, multi-port memory, video direct memory access, dedicated processors, and consequently, consumes many DSP blocks. There is no DSP blocks in our implementation because some functions are performed with LUTs and others are floating point operators. It can been seen in Table 5 that the implementation results in a relatively low hardware complexity since the number of slice LUTs is 12,859 (about 29\% of the device) and the number of slice flip-flops is 13,612 (i.e. $30 \%$ of the device) for the HDR-2 configuration.

Table 4 Design summary.

\begin{tabular}{|c|c|c|c|}
\hline \multicolumn{4}{|c|}{ Clock Domains } \\
\hline Sensor & SDRAM & HDR processing & DVI \\
\hline $114 \mathrm{Mhz}$ & $125 \mathrm{Mhz}$ & $114 \mathrm{Mhz}$ & $25 \mathrm{Mhz}$ \\
\hline \multicolumn{4}{|c|}{ System Parameters } \\
\hline $\mathrm{P}$ & Resolution & Throughput & Framerate \\
\hline 2 & $1,280 \times 1,024$ & 78.6 Mpixels/sec & 60 \\
\hline
\end{tabular}

A significant part of the complexity is due to the MMU$\mathrm{P}$ managing the fine synchronization of the pixel streams between external SDRAM memory and internal BRAMs (about $5 \%$ of the LUTs and $6 \%$ of the flip flops). Only $15.5 \%$ of FIFO / BRAMs are used: BRAMs are mainly used to store the $P$ lines of pixels required by the HDR creating process while FIFO are dedicated to the data synchronization be- 
tween the different steps of the HDR pipeline. The HDR creating and the tone mapping processes consume respectively $9.4 \%$ and $7.7 \%$ of the LUTs and $11.4 \%$ and $9 \%$ of the flip flops due to the implementation of IEEE754 32-bit floating-point arithmetic operators (specifically dividers and multipliers). The entire HDR pipeline process has a global latency of 136 clock rising-edges (i.e. $1.2 \mu \mathrm{s}$ for a $114 \mathrm{MHz}$ system clock). Such a low latency is not perceived by human eye and then, the pipeline process is able to deliver a full HDR video stream at a framerate of $60 \mathrm{fps}$.

Table 5 HDR-2: Summary of hardware synthesis report.

\begin{tabular}{lrcr}
\hline & Used & Available & Utilization \\
\hline Logic utilization & & & \\
Number of Occupied Slices & 5,272 & 11,200 & $47.1 \%$ \\
& & & \\
Complexity distribution & & & \\
Number of Slice LUTs & 12,859 & 44,800 & $28.7 \%$ \\
$\quad$ Memory management & 2,149 & & $4.8 \%$ \\
HDR creating & 4,211 & & $9.4 \%$ \\
Tone mapping & 3,433 & & $7.7 \%$ \\
Post processing & 3,066 & & $6.8 \%$ \\
Number of Slice Flip Flops & 13,612 & 44,800 & $30.4 \%$ \\
Memory management & 2,682 & & $6 \%$ \\
HDR creating & 5,107 & & $11.4 \%$ \\
Tone mapping & 4,045 & & $9 \%$ \\
Post processing & 1,778 & & $4 \%$ \\
Number of FIFO/BRAM & 23 & 148 & $15.5 \%$ \\
\hline
\end{tabular}

The post processing step is also a significant consuming task (7\% of the LUTs and $4 \%$ of the flips flops). This task embeds a dedicated DVI controller designed to display camera data on a LCD monitor. Both live video streams, LDR unprocessed pixel streams and computed HDR video can be displayed without latency noticeable for the viewer. For this purpose, the horizontal and vertical synchronization signals $\left(H_{\text {sync }}\right.$ and $V_{\text {sync }}$ in Fig. 5) are regenerated from the output tone mapped data and used to synchronize pixels by the DVI encoder. Even if this DVI controller consumes significant resources, it can not be considered as a real built-in part of the application. It is only used to stream output data into a LCD monitor where the frames from different stages of the logic (typically, LDR frames after pre-processing and HDR fames after post-processing) can be visually inspected. Thus, ignoring the DVI controller resources, our implementation of the HDR application on the HDR-ARtiSt platform results in a relatively low hardware complexity. This opens the interesting possibility to implement other image processing applications on the FPGA. One of the best candidate applications should be motion analysis and artifacts correction in order to provide a higher quality HDR live video platform.

\section{The HDR-3 video system}

\subsection{HDR-2 limitations}

The HDR-2 system has been evaluated both on images from the Debevec's database (Fig. 10), and on real-scene frames captured by the HDR-ARtiSt platform (Fig. 11).

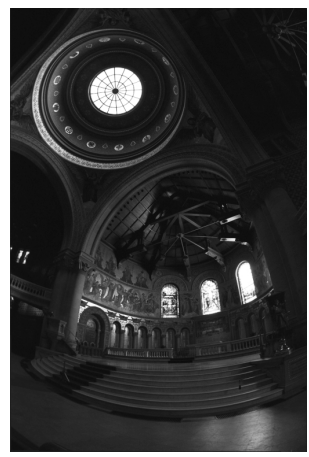

(a) Low exposure

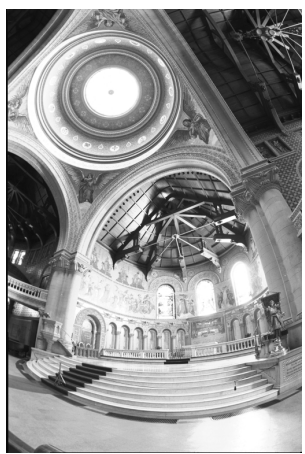

(b) High exposure

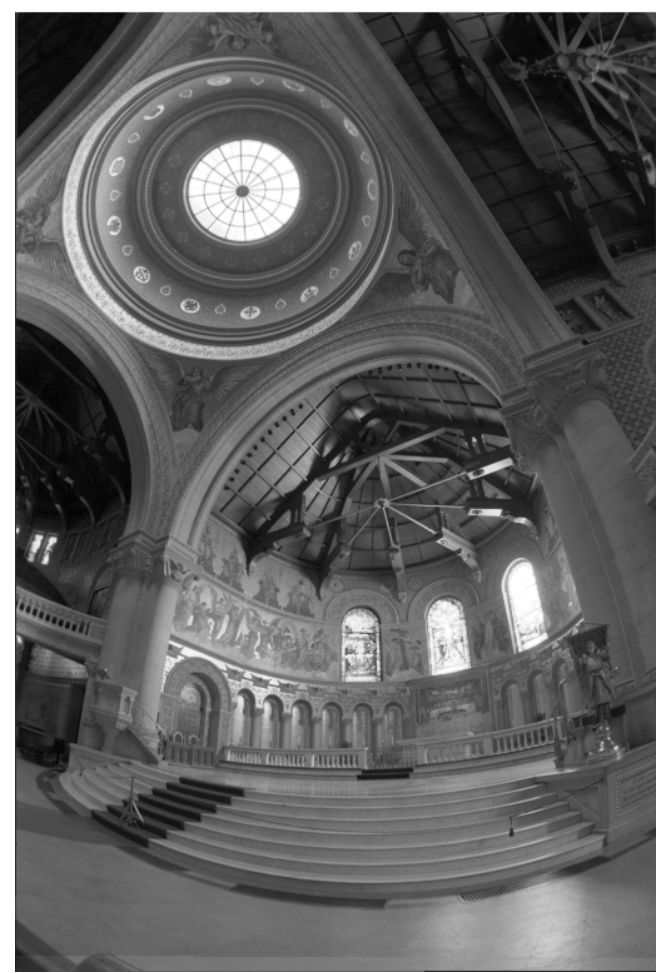

(c) HDR image

Fig. 10 Visual evaluation of the HDR-2 application.

Firstly, the classical database set to test HDR techniques is the Stanford Memorial Church sequence provided by Debevec. It includes a series of images captured with exposure times ranging from $1 / 1,024$ to $32 \mathrm{~s}$. Sets of two images have been selected to evaluate our hardware implementation. Fig. 10 depicts an example of an HDR image built by the HDR2 architecture from a low exposure $\left(\Delta t_{p}=0.5 s\right)$ and a high 
exposure $\left(\Delta t_{p}=16 s\right)$. It should be noted that the exposures are relatively high, due to low-level light conditions inside the church. The tone mapped image reproduces both details in dark and bright areas without noticeable visual artifact.

Secondly, the HDR-2 implementation has been also evaluated with real-world scenes captured by the HDR-ARtiSt platform. The experimental scene is a poorly illuminated desk on which we can find a cup of coffee and some toys (a toy car, a Toy Story figurine, a R2-D2 robot inside the cup of coffee). A bright lamp has been placed behind this scene to significantly enhance the dynamic range (see Fig.11 and Fig. 12). Obviously, the main difficulty for any HDR technique is to simultaneously render the bright area near the light bulb and the dark area inside the cup of coffee, while preserving accuracy on intermediate levels. Note that the exposures are here very low (respectively $0.15 \mathrm{~ms}$ and $1.9 \mathrm{~ms}$ for the low and high exposures) because of the blinding source of light.

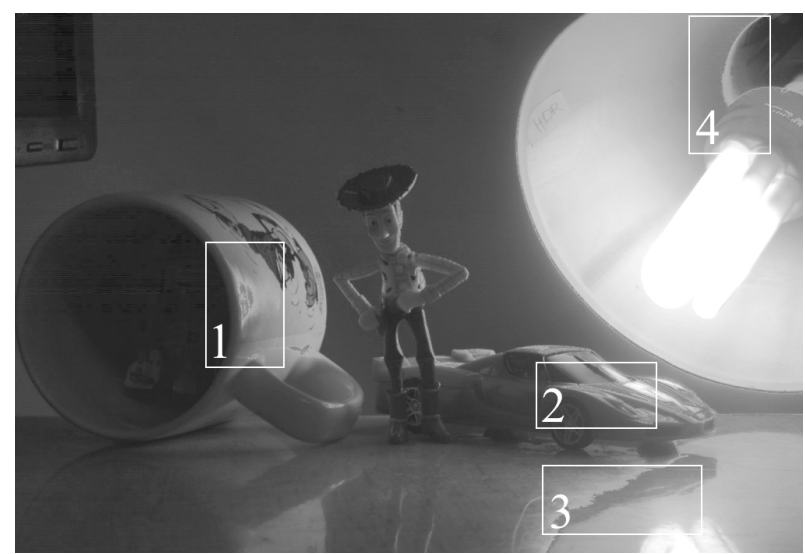

(a) HDR-2 video system limitations in extreme lighting conditions
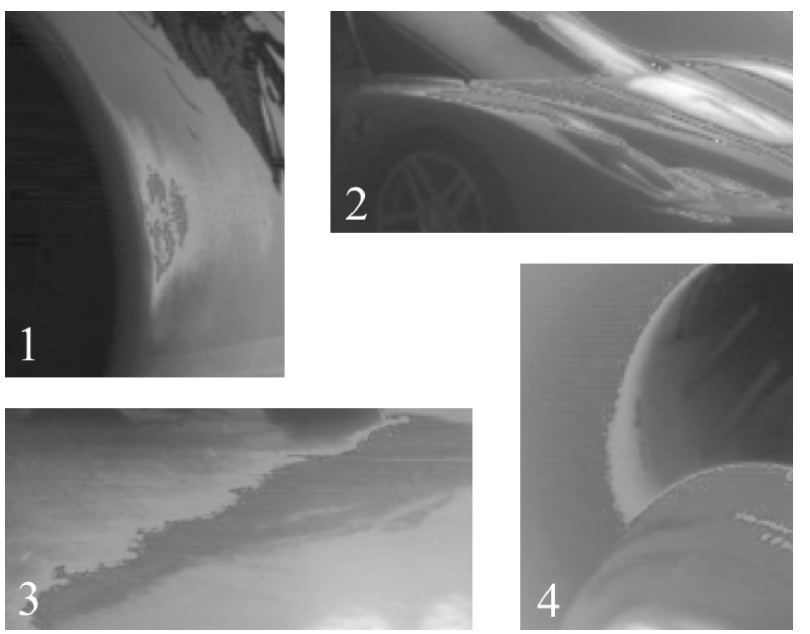

(b) Zoom on different areas

Fig. 11 HDR-2 system limitations in extreme light conditions.

A first visual analysis shows that the results are similar to those obtained previously with the memorial church giving a high-quality reproduction of fine details in dark and bright areas. As examples, the R2D2 figure can be detected even the area inside the cup is particularly dark. On the other side, the word "HDR" written in the lampshade can be read $^{1}$. However, a deeper analysis reveals a lack of details on the medium lightened areas, highlighting some limitations of the HDR-2 implementation. Four different areas have been selected to highlight possible artifacts. These areas are zoomed in and displayed in the bottom part of Fig. 11 , revealing some discontinuities along edges in the cup of coffee (area 1), in the hood of the car (area 2), in the shade of the car (area 3), and in the lampshade (area 4). These observable phenomena are mainly due to the wide dynamic range of the scene exceeding 100,000:1. In such extreme lighting conditions, it seems almost impossible to capture simultaneously information on the light bulb that illuminates the scene, information on the dark part of the scene, and all the intermediate levels with only two frames. Practically, for capturing details in the upper part of the radiance histogram (lamp), the exposure time of the first frame needs to be decreased severely. On the other side, for very dark areas, the exposure time of the second frame is increased drastically. So, with only two captures, it is not possible to capture the whole scene in details. Since the two captures focus on the extreme parts of the illuminance, this implies inevitably a lack of information in the middle area of the histogram, leading to the above-mentioned artifacts.

\subsection{HDR-3 implementation}

To overcome this limitation, the most natural solution is to increase the number of frames. With a complementary middle exposure, the HDR-3 is able to capture the missing data in the median area of the illuminance. However, we limit ourselves to a 3 -frame acquisition process because too many artifacts appear when four or more exposures are used in the case of real scenes with highly moving objects. The HDR-3 appears as the optimal solution able to satisfy the trade-off between high dynamic range with a capture of about $120 \mathrm{~dB}$ dynamic range, low artifacts and high quality.

The middle exposure time $\Delta t_{M}$ is computed from the equation of the exposure value $E V_{M}$ :

$E V_{M}=\log _{2} \frac{\text { f-number }}{2}$ with $E V_{M}=\frac{E V_{L}+E V_{H}}{2}$

where f-number is the aperture. Considering f-number $=1$, $\Delta t_{M}$ can be computed using the following equation:

$\Delta t_{M}=\frac{1}{2^{E V_{M}}}=\frac{1}{2^{\frac{E V_{L}+E V_{H}}{2}}}=\frac{1}{\sqrt{2^{E V_{L}} \times 2^{E V_{H}}}}=\sqrt{\Delta t_{L} \times \Delta t_{H}}$.

The tone mapped frame of the experimental scene using this new implementation is shown in Fig. 12. It is seen that the various artifacts present in Fig. 11 are now widely reduced in this new frame. 


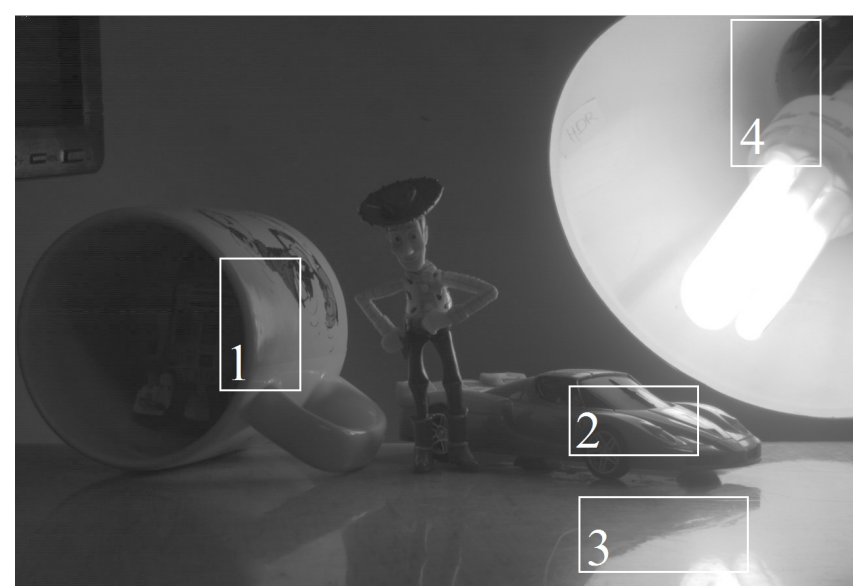

(a) HDR-3 video system improvements in extreme lighting conditions
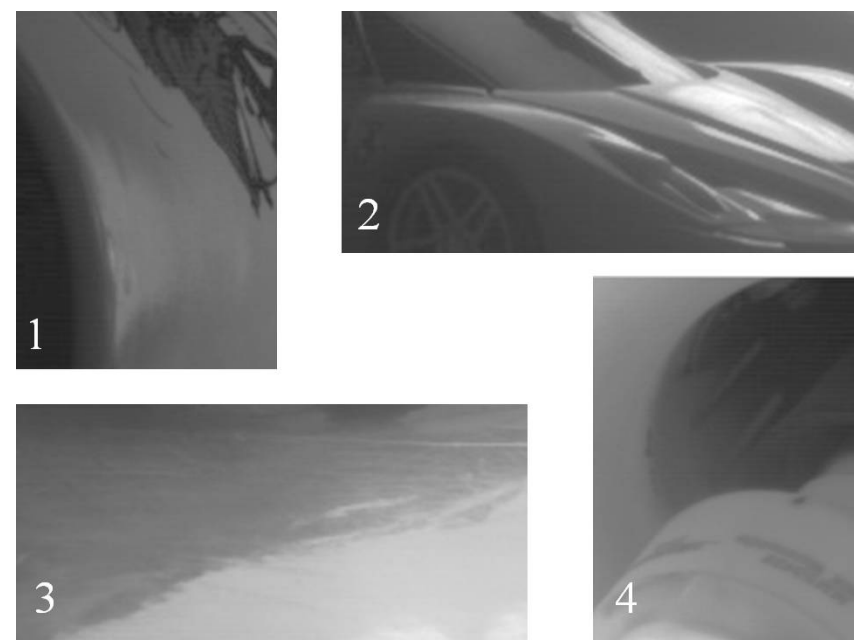

(b) Zoom on different areas

Fig. 12 HDR-3 video system improvements in extreme lighting conditions.

In Table 6, a summary of the hardware complexity of the different blocks is given. The HDR-3 implementation obviously results in a slightly higher complexity than the HDR-2 implementation $(+11.7 \%)$. Among the submodules listed in Table 6 , the amount of the logic resources consumed by the MMU $(+31.6 \%$ in terms of LUTs) and the HDR creating ( $+35.9 \%$ of LUTs and $+11.2 \%$ if flip flops) are increased. Moreover, when the number of frames raises from 2 to 3, there is a significant rise in the usage of block RAMs $(+30.4 \%)$, mainly used by the MMU. In contrast to these significant increases, the tone mapping module does not undergo any change and consumes the same resources. The other modules of the system are relatively less affected, except the number of flip flops used by the post processing submodule. Indeed, this module embeds some additional resources (registers, multiplexers for example) to manage the 3 LDR and the HDR streams and route them to the DVI output.

\footnotetext{
1 The R2D2 figure and the word "HDR " are only visible in elec-
} tronic version of the paper and are almost hidden in the print version.
Table 6 HDR-3: Summary of hardware synthesis report.

\begin{tabular}{lrrr}
\hline & Used & Utilization & $\begin{array}{r}\text { Variation } \\
\text { with HDR-2 }\end{array}$ \\
\hline $\begin{array}{lrrr}\text { Logic utilization } \\
\text { Number of Occupied Slices }\end{array}$ & 5,891 & $52.6 \%$ & $+11.7 \%$ \\
Complexity distribution & & & \\
Number of Slice LUTs & 15,281 & $34.11 \%$ & $+18.8 \%$ \\
$\quad$ Memory management & 2,829 & $6.3 \%$ & $+31.6 \%$ \\
HDR creating & 5,722 & $12.8 \%$ & $+35.9 \%$ \\
Tone mapping & 3,433 & $7.7 \%$ & $0 \%$ \\
Post processing & 3,297 & $7.4 \%$ & $+7.5 \%$ \\
Number of Slice Flip Flops & 15,134 & $33.8 \%$ & $+11.2 \%$ \\
Memory management & 2,682 & $6 \%$ & $+0.1 \%$ \\
HDR creating & 6,071 & $13.5 \%$ & $+18.9 \%$ \\
Tone mapping & 4,045 & $9 \%$ & $0 \%$ \\
Post processing & 2,333 & $5.2 \%$ & $+31.2 \%$ \\
Number of FIFO/BRAMs & 30 & $20.3 \%$ & $+30.4 \%$ \\
\hline
\end{tabular}

\subsection{Extending the architecture to color data}

The current B\&W HDR-ARtiSt platform could be easily enhanced with a color sensor. In a traditional HDR workflow, HDR creating, tone mapping and other rendering steps are applied after demosaicing. However, as explained in Tamburrino et al [45], the retinal processing of the human visual system (HVS) performs most of adaptation operations on the cone mosaic before demosaicing. According to this principle, Tamburrino et al. [45] proposes a new HDR workflow in which the tone mapping are applied directly on the color filter array (CFA) image, instead of the already demosaiced image. Demosaicing is then the last step of the HDR workflow. Such an approach is then directly compatible with the HDR pipeline implemented on the HDR-ARtiSt platform. Indeed, the HDR creating and the tone mapping operation can be applied on the pixels of the mosaiced image using the three different response curves of the sensor (for the red, green and blue channels), before applying demosaicing. In terms of computation, it does not increase computational complexity because there is only one RAW data stream to process and not three RGB streams. The demosaicing step, which converts the RAW to RGB data, is handled by a specific hardware block (named "Color Filter Array Interpolation") provided by Xilinx. This block generates the missing color components associated with the commonly used Bayer pattern in digital camera systems. Additionally, a white balance algorithm IP provided by Xilinx can also be implemented in our platform in order to render colors as natural as possible. In terms of hardware resources on the HDRARtiSt platform, it only requires two more LUTs to store the response curves and the demosaicing/white balance operators provided by Xilinx. According to Debevec and Malik [9], we can also, for simplicity, use an identical single curve for both $\mathrm{R}$ and $\mathrm{G}$ components because these two response curves are very consistent. 


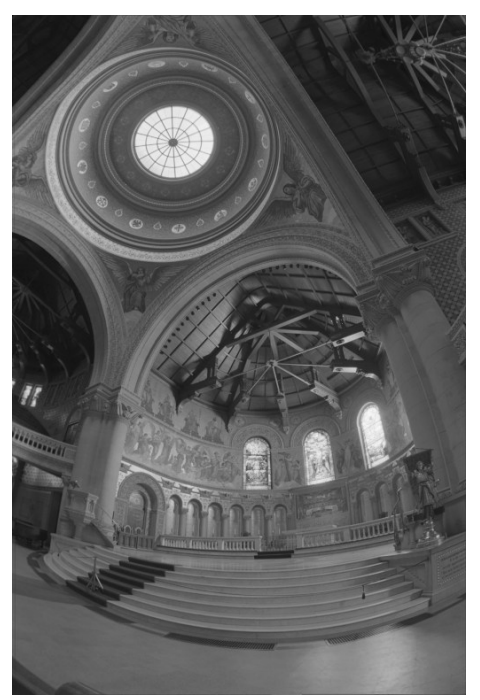

(a) Gold standard

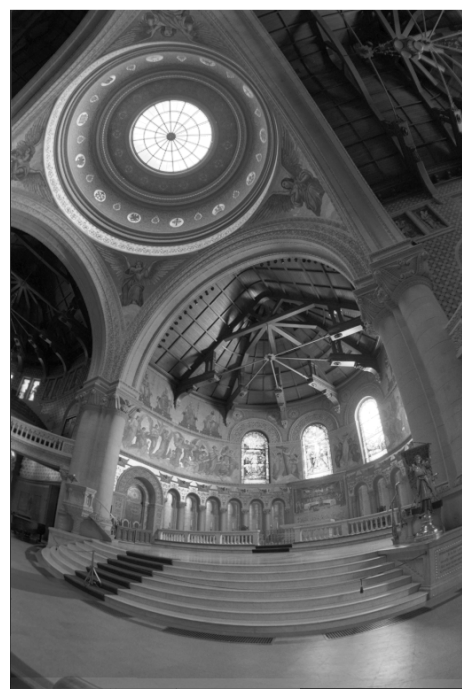

(b) Our system.

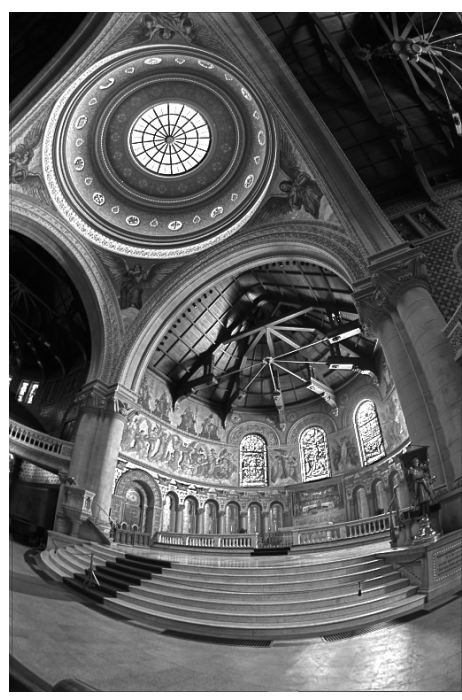

(c) Hassan et al. [19]

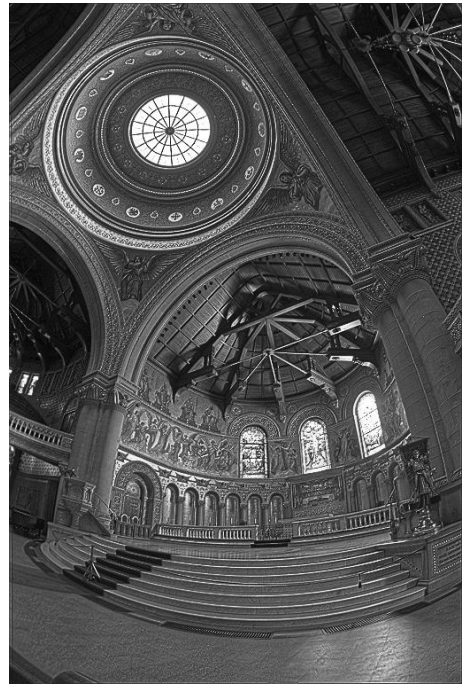

(d) Vytla et al. [48]

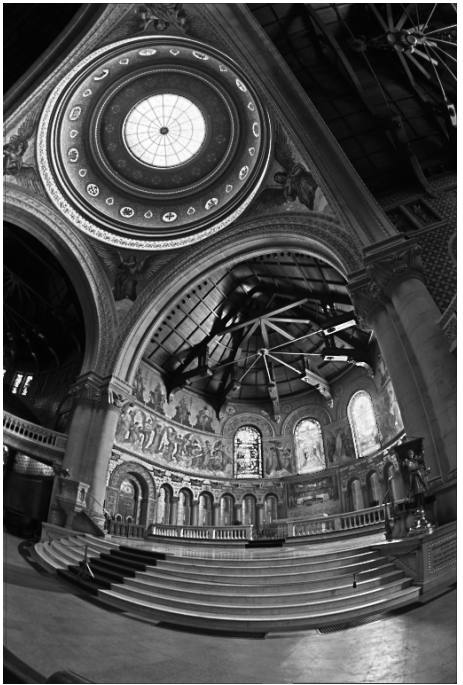

(e) Slomp et al. [44]

Fig. 13 Comparison of the proposed system output with different real-time tone mapping methods.

\subsection{Comparison with state-of-the-art existing architectures}

In addition to performance results, a comparison of the HDR3 architecture with three other systems has been conducted. Two of them are on a FPGA-based architecture $[19,48]$ and the last one on a GPU platform [44]. However, they implement only the tone mapping, using standard HDR images as inputs. Indeed, hardware vision systems for capturing and rendering HDR live video are at a adolescence stage [31] and we failed to find in the literature a full HDR system implementing all the steps from capturing to rendering. The HDR-3 architecture, on the other hand, is an innovative camera prototype implementing the full HDR pipeline. From this purely algorithmic point of view, our real-time implementation outstrips the computational capabilities of the three other implementations.
Table 7 summarizes the comparison results in terms of processing performance (fps, resolution). From a raw performance point of view, our architecture runs at $60 \mathrm{fps}$ on a $1.280 \times 1.024$-pixel resolution, giving an overall throughput of 78.6 megapixels per second. This performance is significantly higher than the two other FPGA-based architectures and lower than the GPU implementation. Nevertheless, the Nvidia 8800 GTX processor used in this GPU alternative is not well suited to low-power embedded systems such as smart cameras.

We have also compared the image quality of the tonemapped images produced by the different architectures using five metrics: Mean Square Error (MSE), Normalized Root Mean Square Error (NRMSE), Peak Signal-to-Noise Ratio (PSNR), Universal Quality Index (UQI [49]), and the Structural SIMilarity (SSIM [50]). UQI roughly corresponds to the human perception of distance among images. The value 
Table 7 Performance comparison with existing real-time tone mapping implementations.

\begin{tabular}{|c|c|c|c|c|}
\hline & This work & \begin{tabular}{|l} 
FPGA \\
{$[19]$}
\end{tabular} & \begin{tabular}{|l} 
FPGA \\
{$[48]$}
\end{tabular} & $\begin{array}{l}\text { GPU } \\
\text { [44] }\end{array}$ \\
\hline & Virtex 5 & $\begin{array}{l}\text { Stratix II } \\
\text { EP2S130F780 }\end{array}$ & \begin{tabular}{|l|} 
Stratix II \\
EP2S15F484 \\
GO
\end{tabular} & $\begin{array}{l}\text { Nvidia GeForce } \\
8800 \text { GTX }\end{array}$ \\
\hline Frame rate & 60 & & & 100 \\
\hline $\begin{array}{l}\text { Frame } \\
\text { size } \\
\text { (pixels) }\end{array}$ & $1,280 \times 1,024$ & $1,024 \times 768$ & $1,024 \times 1,024$ & $1,024 \times 1,024$ \\
\hline MSE & $2.8695 \times 10^{2}$ & $6.6801 \times 10^{2}$ & $3.8646 \times 10^{2}$ & $5.8607 \times 10^{2}$ \\
\hline NRMSE & $1.7 \times 10^{-1}$ & $3.8 \times 10^{-1}$ & $1.3 \times 10^{-1}$ & $1.9 \times 10^{-1}$ \\
\hline $\begin{array}{l}\text { PSNR } \\
(\mathrm{dB})\end{array}$ & $2.355 \times 10^{1}$ & $1.988 \times 10^{1}$ & $2.226 \times 10^{1}$ & $2.045 \times 10^{1}$ \\
\hline $\begin{array}{l}\text { SSIM } \\
\text { UQI }\end{array}$ & $\begin{array}{l}9.3 \times 10^{-1} \\
0.90\end{array}$ & $\begin{array}{l}6.9 \times 10^{-1} \\
0.71\end{array}$ & $\begin{array}{l}5.5 \times 10^{-1} \\
0.67\end{array}$ & $\begin{array}{l}7.0 \times 10^{-1} \\
0.70\end{array}$ \\
\hline
\end{tabular}

of the UQI between two images is in the $[-1,1]$ range, and is 1 for identical images, 0 for uncorrelated images, and -1 for completely anticorrelated images. The Structural SIMilarity (SSIM) index is a method for measuring the similarity between two images. The SSIM index can be viewed as a quality measure of one of the images being compared, provided the other image is regarded as of perfect quality. It is an improved version of the UQI index.

Our gold standard is the tone-mapped image of the Stanford memorial church with the method by F. Drago and Chiba [13]. This technique is described as the most natural method and also the most detailed method in dark region [51]. Considering the gold standard to be the reference signal, and the difference between this reference and the images processed by the other architectures to be the noise, the MSE, the PSNR and the UQI have been calculated for each architecture. The set of images is shown in Fig. 13. The method from Hassan and Carletta [19] seems to provide more contrast and image details than our proposed method. This is mainly due to the fact they use as inputs HDR images with 28 bits per pixel, obtained off-line from a set of 16 multiple exposures of the same scene. However, the visual evaluation of the different images reveals that the HDR-3 system gives comparable results to the gold standard. This visual feeling is reinforced by different quality metrics. The HDR-3 architecture has the lowest MSE, the lowest NRMSE and the highest PSNR. Moreover, with an UQI value of 0.90 and a SSIM of 0.93 , this indicates that the HDR images provided by our architecture are the closest from the gold standard and then, that the visual rendering of these images can be considered as the most natural one.

\section{Conclusion}

In this paper, we present a complete hardware vision system called HDR-ARtiSt based on a standard image sensor associated with a FPGA development board. This smart camera dedicated to real-time HDR live video fulfils drastic realtime constraints while satisfying image quality requirements. It embeds a full HDR pipeline, doing successively multiple captures, HDR creating, tone mapping, and streaming of the HDR live video to a LCD monitor.

The HDR-ARtist system has been built as an adaptive platform. From a purely hardware point of view, our HDR pipeline can be easily adapted to many conventional CMOS sensors. Using a new sensor only requires the design of a specific sensor board to be plugged onto the FPGA motherboard and the offline evaluation of the camera transfer function for the new sensor. From an application point of view, the HDR pipeline can be parametrized in terms of number of frames, depending on the scene dynamic range. In this paper, two different real-time implementations, using respectively 2 and 3 frames have been discussed. For each implementation, we obtain high-performance results due to a finely tuned implementation of the original Debevec's algorithm and a global tone mapping from Duan. The comparison with other state-of-the-art architectures highlights a high visual quality, close to Drago's algorithm, known as one of the best tone mappers. Moreover, to achieve high temporal performance, the HDR-ARtiSt platform embeds a dedicated memory management unit. This memory unit has been specifically designed for managing multiple parallel video streams to feed the HDR creating process. It significantly contributes to the whole performance of the system. Indeed, the HDR pipeline is synchronized on the image sensor framerate. It builds a new HDR frame for each new sensor capture and delivers a live stream of displayable content with a bandwidth of 629 Mbits/s. Such a memory unit also limits motion blur and ghosting artifacts in the HDR frames because of the continuous acquisition process.

All these results open interesting avenues for future exploration both on hardware and software issues. New releases of the HDR-ARtist platform embedding FPGA from Xilinx Virtex-6 and Virtex-7 families are currently in development. They will give us opportunity to implement the most recent algorithms for computing radiance maps, and for local/global tone mapping. We also plan to automatically evaluate the real dynamic range of the scene and then to dynamically adapt the number of required captures to build the best HDR frame.

Finally, HDR video is prone to ghosting artifacts, which can appear with motion in the scene during the multiple captures. So, we intend to study and implement onto the FPGA dedicated ghost detection techniques in order to provide a real-time ghost-free HDR live video. A hw/sw approach based on reprogrammable Zynq architecture is under construction, which will simplify certain operations, and help to add some patches as the correction of local movements.

Acknowledgements This work was supported by the DGCIS (French Ministry for Industry) within the framework of the European HiDRaLoN project and by grants from the Conseil Regional de Bourgogne. 


\section{References}

1. Acosta-Serafini P, Ichiro M, Sodini C (2004) A 1/3" VGA linear wide dynamic range CMOS image sensor implementing a predictive multiple sampling algorithm with overlapping integration intervals. IEEE Journal of Solid-State Circuits 39(9):1487-1496

2. Akil M, Grandpierre T, Perroton L (2012) Real-time dynamic tone-mapping operator on gpu. Journal of RealTime Image Processing 7(3):165-172

3. Akyuz A (2012) High dynamic range imaging pipeline on the gpu. Journal of Real-Time Image Processing pp 1-15, DOI 10.1007/s11554-012-0270-9

4. Alston L, Levinstone D, Plummer W (1987) Exposure control system for an electronic imaging camera having increased dynamic range

5. Boschetti A, Adami N, Leonardi R, Okuda M (2011) An optimal Video-Surveillance approach for HDR videos tone mapping. In: 19th European Signal Processing Conference EUSIPCO 2011, Barcelona

6. Čadík M, Wimmer M, Neumann L, Artusi A (2008) Evaluation of HDR tone mapping methods using essential perceptual attributes. Computers \& Graphics 32:330-349

7. Cembrano G, Rodriguez-Vazquez A, Galan R, JimenezGarrido F, Espejo S, Dominguez-Castro R (2004) A $1000 \mathrm{fps}$ at $128 \times 128$ vision processor with 8-bit digitized I/O. IEEE Journal of Solid-State Circuits 39(7):1044-1055

8. Chiu CT, Wang TH, Ke WM, Chuang CY, Huang JS, Wong WS, Tsay RS, Wu CJ (2010) Real-time tonemapping processor with integrated photographic and gradient compression using $0.13 \mu \mathrm{m}$ technology on an ARM SoC platform. Journal of Signal Processing Systems 64(1):93-107

9. Debevec PE, Malik J (1997) Recovering high dynamic range radiance maps from photographs. In: Proceedings of the 24th annual conference on Computer graphics and interactive techniques (SIGGRAPH), pp 369-378

10. Devlin K, Chalmers A, Wilkie A, Purgathofer W (2002) Tone reproduction and physically based spectral rendering. In: Eurographics 2002, Eurographics Association, pp 101-123

11. Duan J, Bressan M, Dance C, Qiu G (2010) Tonemapping high dynamic range images by novel histogram adjustment. Pattern Recognition 43:1847-1862

12. Dubois J, Ginhac D, Paindavoine M, Heyrman B (2008) A $10000 \mathrm{fps}$ CMOS sensor with massively parallel image processing. IEEE Journal of Solid-State Circuits 43(3):706-717

13. F Drago TA K Myszkowski, Chiba N (2003) Adaptive logarithmic mapping for displaying high contrast scenes. EUROGRAPHICS 2003 / P Brunet and D Fellner Volume 22 (2003), Number 3(3): $x x-x x$

14. Fattal R, Lischinski D, Werman M (2002) Gradient domain high dynamic range compression. ACM Transactions on Graphics (TOG) 21:249-256
15. G Yourganov WS (2001) Acquiring high dynamic range video at video rates. Tech. rep., Dept. of Computer Science, York University

16. Gelfand N, Adams A, Park SH, Pulli K (2010) Multiexposure imaging on mobile devices. In: Proceedings of the international conference on Multimedia, New York, USA, pp 823-826

17. Graf HG, Harendt C, Engelhardt T, Scherjon C, Warkentin K, Richter H, Burghartz J (2009) High dynamic range cmos imager technologies for biomedical applications. Solid-State Circuits, IEEE Journal of 44(1):281 -289

18. Granados M, Ajdin B, Wand M, Theobalt C, Seidel H, Lensch H (2010) Optimal HDR reconstruction with linear digital cameras. In: IEEE Conference on Computer Vision and Pattern Recognition (CVPR), pp 215-222

19. Hassan F, Carletta J (2007) A real-time FPGAbased architecture for a Reinhard-like tone mapping operator. In: Proceedings of the 22nd ACM SIGGRAPH/EUROGRAPHICS symposium on Graphics hardware, Aire-la-Ville, Switzerland, pp 65-71

20. Hrabar S, Corke P, Bosse M (2009) High dynamic range stereo vision for outdoor mobile robotics. In: Robotics and Automation, 2009. ICRA '09. IEEE International Conference on, pp $430-435$

21. Kang SB, Uyttendaele M, Winder S, Szeliski R (2003) High dynamic range video. Tech. rep., Interactive Visual Media Group, Microsoft Research, Redmond, WA

22. Kavusi S, El Gamal A (2004) A quantitative study of high dynamic range image sensor architectures. In: Proceedings of the SPIE Electronic Imaging '04 Conference, vol 5301, pp 264-275

23. Ke WM, Wang TH, Chiu CT (2009) Hardware-efficient virtual high dynamic range image reproduction. In: Proceedings of the 16th IEEE International Conference on Image Processing (ICIP'09), Piscataway, NJ, USA, pp 2665-2668

24. Lee SH, Woo H, kang MG (2010) Global illumination invariant object detection with level set based bimodal segmentation. Circuits and Systems for Video Technology, IEEE Transactions on 20(4):616-620

25. Leflar M, Hesham O, Joslin C (2009) Use of high dynamic range images for improved medical simulations. In: Magnenat-Thalmann N (ed) Modelling the Physiological Human, Lecture Notes in Computer Science, vol 5903, Springer Berlin Heidelberg, pp 199-208

26. Lindgren L, Melander J, Johansson R, Moller B (2005) A multiresolution 100-GOPS 4-Gpixels/s programmable smart vision sensor for multisense imaging. IEEE Journal of Solid-State Circuits 40(6):1350-1359

27. Liu J, Hassan F, Carletta J (2013) A study of hardwarefriendly methods for gradient domain tone mapping of high dynamic range images. Journal of Real-Time Image Processing pp 1-17, DOI 10.1007/s11554-0130365-y

28. Mangiat S, Gibson J (2011) Inexpensive high dynamic range video for large scale security and surveillance. 
In: Military Communications Conference, 2011 - MILCOM 2011, pp $1772-1777$

29. Mitsunaga T, Nayar S (1999) Radiometric self calibration. In: IEEE Conference on Computer Vision and Pattern Recognition (CVPR), vol 1, pp 374-380

30. Morfu S, Marquié P, Nofiélé B, Ginhac D (2008) Nonlinear systems for image processing. In: Hawkes PW (ed) Advances in Imaging and Electron Physics, vol 152, Elsevier, pp $79-151$

31. Orazio Gallo WCC Natasha Gelfandz, Pulli K (2009) Artifact-free high dynamic range imaging. Tech. rep., University of California

32. Pattanaik SN, Tumblin J, Yee H, Greenberg DP (2000) Time-dependent visual adaptation for fast realistic image display. In: Proceedings of the 27th annual conference on Computer graphics and interactive techniques, ACM Press/Addison-Wesley Publishing Co., New York, NY, USA, SIGGRAPH '00, pp 47-54

33. Reinhard E, Devlin K (2005) Dynamic range reduction inspired by photoreceptor physiology. IEEE Transactions on Visualization and Computer Graphics 11:13 24

34. Reinhard E, Stark M, Shirley P, Ferwerda J (2002) Photographic tone reproduction for digital images. ACM Transactions on Graphics (TOG) 21(3):267-276

35. Reinhard E, Stark M, Shirley P, Ferwerda J (2002) Photographic tone reproduction for digital images. ACM Transactions on Graphics 21(3):267-276

36. Reinhard E, Ward G, Pattanaik S, Debevec P, Heidrich W, Myszkowski K (2010) High Dynamic Range Imaging: Acquisition, Display, and Image-based Lighting, 2nd edn. The Morgan Kaufmann series in Computer Graphics, Elsevier, Burlington, MA

37. Robertson MA, Borman S, Stevenson RL (2003) Estimation-theoretic approach to dynamic range enhancement using multiple exposures. Journal of Electronic Imaging 12(2):219-228

38. Ruedi PF, Heim P, Gyger S, Kaess F, Arm C, Caseiro R, Nagel JL, Todeschini S (2009) An SoC combining a 132dB QVGA pixel array and a 32b DSP/MCU processor for vision applications. In: Solid-State Circuits Conference - Digest of Technical Papers, 2009. ISSCC 2009. IEEE International, pp 46-47,47a

39. Sakakibara M, Kawahito S, Handoko D, Nakamura N, Higashi M, Mabuchi K, Sumi H (2005) A highsensitivity CMOS image sensor with gain-adaptative column amplifiers. IEEE Journal of Solid-State Circuits 40(5):1147-1156

40. Schanz M, Nitta C, Bussmann A, Hosticka B, Wertheimer R (2000) A high-dynamic-range cmos image sensor for automotive applications. Solid-State Circuits, IEEE Journal of 35(7):932 -938

41. Schlick C (1995) Quantization techniques for visualization of high dynamic range pictures. In: Sakas G, Maller S, Shirley P (eds) Photorealistic Rendering Techniques, Focus on Computer Graphics, Springer Berlin Heidelberg, pp 7-20
42. Schubert F, Schertler K, Mikolajczyk K (2009) A handson approach to high-dynamic-range and superresolution fusion. In: IEEE Workshop on Applications of Computer Vision (WACV), pp 1-8

43. Sérot J, Ginhac D, Chapuis R, Dérutin JP (2001) Fast prototyping of parallel-vision applications using functional skeletons. Machine Vision and Applications 12:271-290

44. Slomp M, Oliveira MM (2008) Real-time photographic local tone reproduction using summed-area tables. In: Computer Graphics International, pp 82-91

45. Tamburrino D, Alleysson D, Meylan L, Suesstrunk S (2008) Digital camera workflow for high dynamic range images using a model of retinal processing. In: DiCarlo J, Rodricks B (eds) Digital Photography IV, Proceedings of SPIE, vol 6817, Conference on Digital Photography IV, San Jose, CA, JAN 28-29, 2008

46. e2v technologies (2009) Ev76c560 BW and colour CMOS sensor. URL http://www.e2v.com/products-andservices/high-performance-imaging-solutions/imagingsolutions-cmos-ccd-emccd/

47. Tocci MD, Kiser C, Tocci N, Sen P (2011) A versatile hdr video production system. ACM Trans Graph 30(4):41:1-41:10

48. Vytla L, Hassan F, Carletta J (2013) A real-time implementation of gradient domain high dynamic range compression using a local poisson solver. Journal of RealTime Image Processing 8(2):153-167

49. Wang Z, Bovik AC (2002) A universal image quality index. Signal Processing Letters, IEEE 9(3):81-84

50. Wang Z, Bovik A, Sheikh H, Simoncelli E (2004) Image quality assessment: from error visibility to structural similarity. Image Processing, IEEE Transactions on 13(4):600-612

51. Yoshida A, Blanz V, Myszkowski K, Seidel HP (2005) Perceptual evaluation of tone mapping operators with real-world scenes. In: Human Vision \& Electronic Imaging X, SPIE, pp 192-203

52. Youm SJ, ho Cho W, Hong KS (2005) High dynamic range video through fusion of exposured-controlled frames. In: Proceedings of IAPR Conference on Machine Vision Applications, pp 546-549 